\title{
Fluctuations in the Diffuse Soft X-ray Background; Probably of Galactic Halo Origin
}

\author{
T.J. Sumner, S.D. Sidher, J.J. Quenby, M. Hernandez, A. Mian, R. Jesseit, \\ and M. Gambhir
}

Astrophysics Group, Blackett Laboratory, Imperial College, Prince Consort Road, London, SW7 2BZ, UK

\begin{abstract}
Soft X-ray background data from the ROSAT PSPC have been fitted by a model including emission from the local bubble, embedded hot gas within the disk, hot gas in the Galactic halo and extra-galactic emission, together with local and disk absorption. In all directions a halo component at $10^{6.2} \mathrm{~K}(0.2 \mathrm{keV})$ is required for an acceptable fit. The halo emission measure shows spatial variability and the global trend suggests a disk-like planar rather than more extended spherical distribution. New values for the emission measure within the local bubble are derived.
\end{abstract}

\section{Introduction}

Detailed spectral modelling is being used to establish the mean and fluctuating components of the local bubble, embedded disk and halo contributions to the soft X-ray background (Sidher et al. 1996). ROSAT shadowing data (Burrows and Mendenhall, 1991, Snowden et al. 1991) have cast doubts on early models of the $<2 \mathrm{keV}$ background, based either on absorption of a $3 \times 10^{6} \mathrm{~K}$ halo or on purely local bubble emission with cavity walls at varying distances. Sidher et al. 1996 showed that the minimal model giving an acceptable spectral fit must include an extra-galactic power law component, a hot gas Galactic halo component at $\log T=6.2$ and a local bubble at $\log T=5.9$. In some directions, such as the Lockman hole region (Sidher et al. 1997), an additional component is needed which may indicate embedded disk emission from wind or supernova driven bubbles. Radio data and cosmic ray propagation studies suggest an extended galactic halo and a sufficiently hot and extensive gas component is needed for stability (Bloemen 1987). Kahn, 1994 reviews the fountain model in which regions of upward flow, driven by SNRs, are balanced by others returning to the disk. Cosmic rays may drive the flow far out (eg Breitschwerdt et al., 1993). The $0.5-2 \mathrm{keV}$ soft X-ray spatial autocorrelation function has been used to study the contribution of various types of source to the extra-galactic background (eg Soltan et al. 1996, Barcons and Fabian, 1989). The possibility of substantial Galaxy related spatial fluctuation has not been considered. Suto et al. (1996) show that a Local Group halo origin for the $1 \mathrm{keV}$ background could account for the microwave background quadrupole anisotropy through the Sunyaev-Zel'dovich effect, although Pildis and McGaugh, 1996 disagree using data from other poor galaxy groups. 


\section{$2 \quad$ Spectral Modelling}

Modelling is done using any number of concentric shells, each of which may contain a mixture of hot emitting gas and cold absorber. Details may be found in Sidher et al. 1996. The radiative power loss function of Landini and Monsignori Fossi, 1990 is used for all emitting components except the extragalactic background where a power law is used. Predicted spectra are convolved with the instrument performance. 'Clean' observational data are extracted from archived ROSAT PSPC data using a procedure designed to minimize contamination from particle backgrounds. From these, images are displayed and binned background spectra are recovered from within regions devoid of obvious sources. Observed and predicted spectra, identically binned, are then using a $\chi^{2}$ test. Best fit models and their associated free parameter ranges are assessed using the criteria of Lampton et al., 1976.

\section{Results and Discussion}

\subsection{Local Bubble and Galactic Halo Emission Measure}

Fits have been performed on 78 ROSAT observations including the Draco complex, the Lockman hole and M87 (Sumner et al. 1997). For the local bubble a fixed temperature $(\log T=5.9$, Lieu et al., 1992) has been used for all directions and the best fit emission measures (EMs) are shown on Fig. 1. The radius of each circle is proportional to the EM which ranges from 0.0028 to $0.011 \mathrm{~cm}^{-6} \mathrm{pc}$. The mean value of all pointings is $0.006 \mathrm{~cm}^{-6} \mathrm{pc}$. The temperature and EM of the halo component are free parameters in the fits. Apart from the M87 region, the best fit halo temperature is constant (to within the fitting step size of $\Delta \log T$ ) across the sky and is given by $\log T=6.2$. The best fit EMs are shown in Fig. 2. The radius of each circle is proportional to the EM which ranges from 0.0096 to $0.083 \mathrm{~cm}^{-6} \mathrm{pc}$. The mean value of all pointings is $0.024 \mathrm{~cm}^{-6} \mathrm{pc}$. There is a tendency for the EM to increase towards the galactic equator, which we have previously shown (Sidher et al. 1996) is consistent with a disk-like distribution (following Bloemen 1987).

\subsection{Local Group Halo Model}

In Fig. 3 solid contours show the expected variation in halo EM assuming it has a spherical distribution about the Local Group centre with a density profile following a speculative dark matter distribution (see model of Sidher et al. 1996 with $p=2$ ). Contours labels are in units of $0.0032 \mathrm{~cm}^{-6} \mathrm{pc}$. The model predicts an decrease in EM in all directions away from the centre of the Local Group. This trend is not consistent with the results. 
Local Bubble Emission Measure Variation $90^{\circ}$

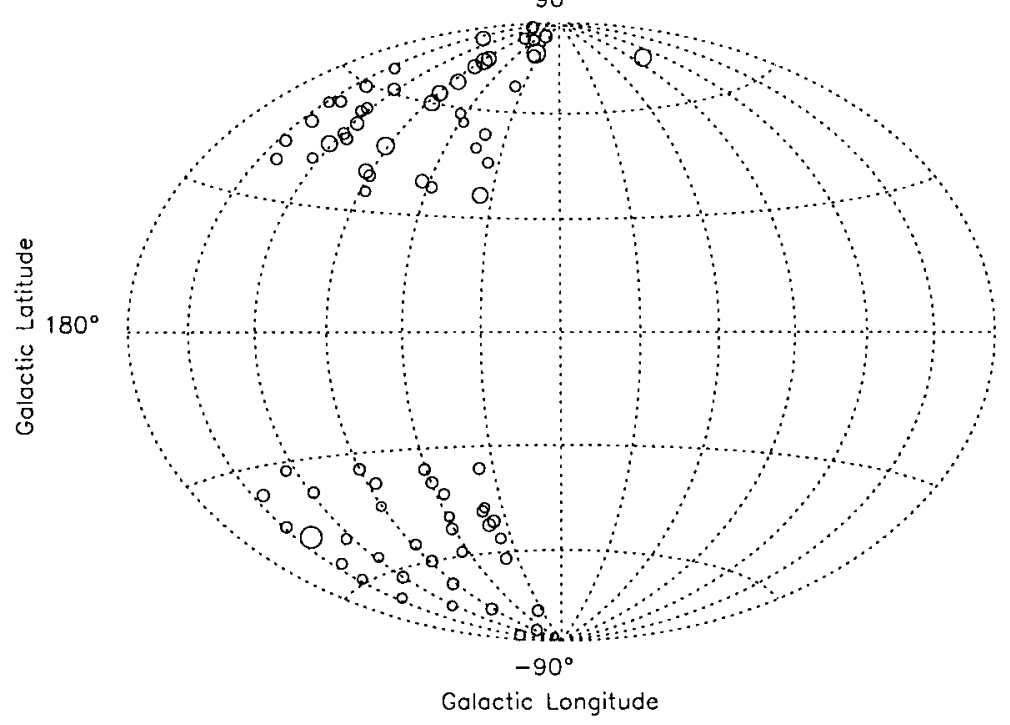

$180^{\circ}$

Fig. 1. Local Bubble emission measures

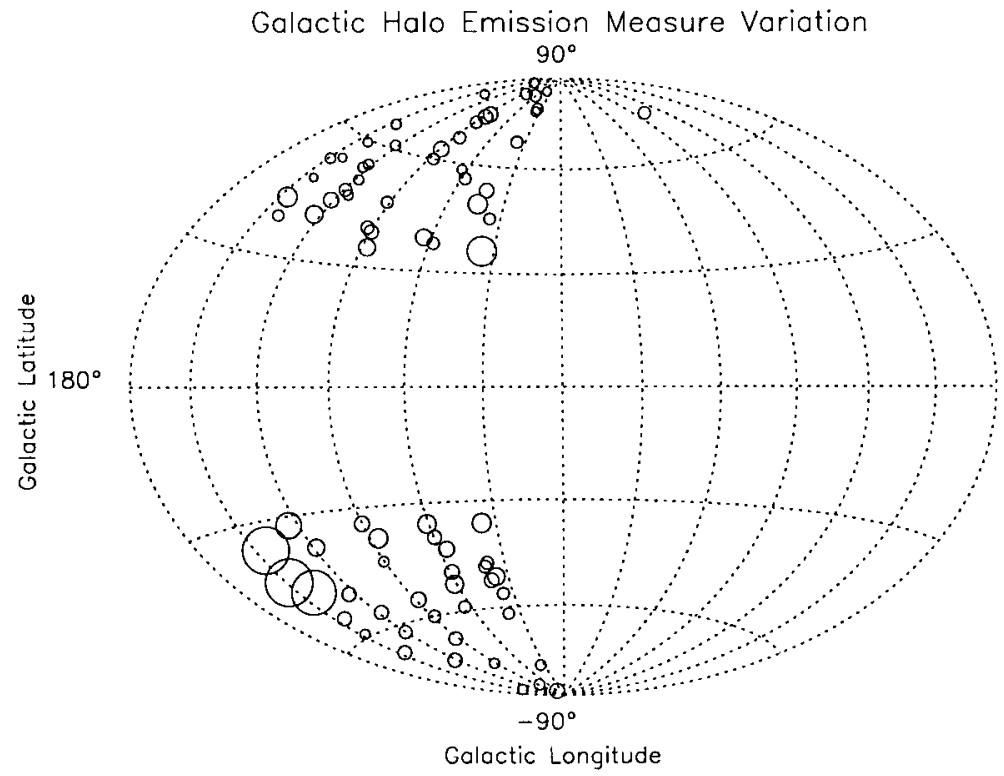

Fig. 2. Galactic Halo emission measures 


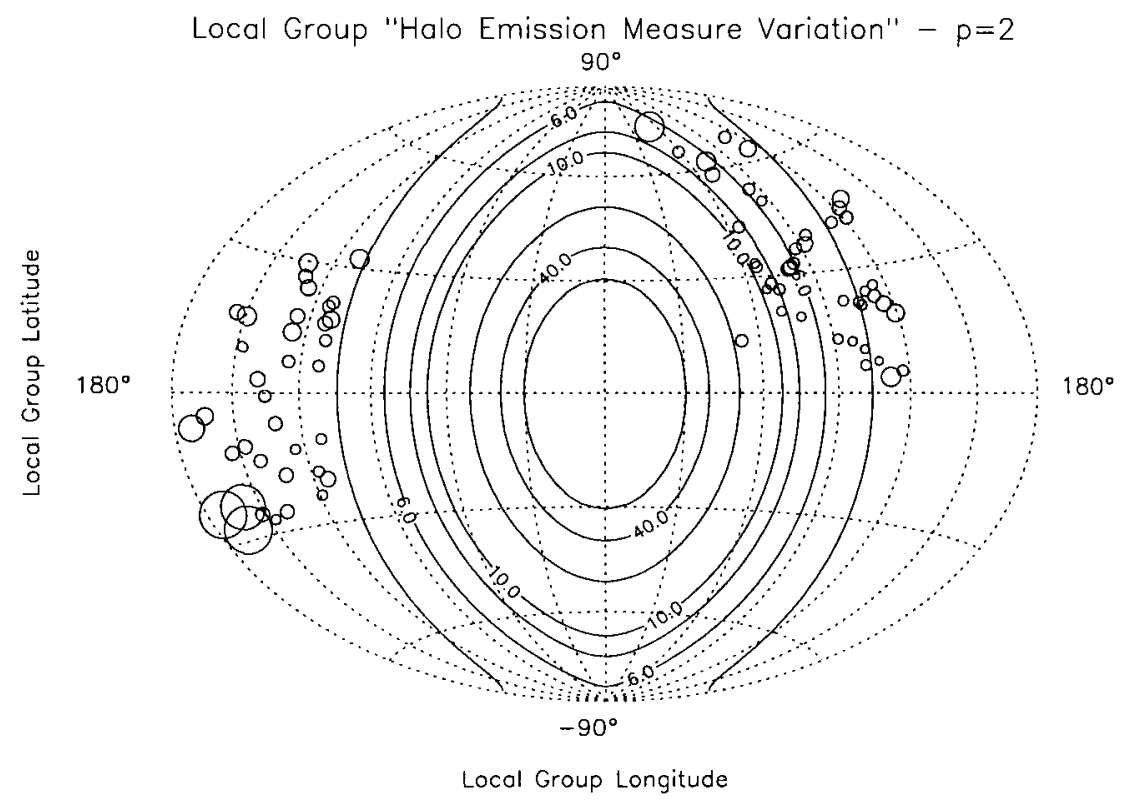

Fig. 3. Local Group Halo emission measure

\section{References}

Barcons X., \& Fabian A.C. (1989): MNRAS 237, 119

Bloemen J.B.G.M. (1987): ApJ 322, 694

Breitschwerdt D., McKenzie J.F., \& Völk H.J. (1993): A\&A 269, 54

Burrows D.N., \& Mendenhall J.A. (1991): Nature 351, 629

Kahn F.D. (1994): Ap\&SS 216, 325

Lampton M., Margon B., \& Bowyer S. (1976): ApJ 208, 177

Landini M., \& Monsignori Fossi B.C. (1990): A\&A 82, 229

Lieu R., Quenby J.J., Sidher S.D., Sumner T.J., Willingale R., West R.G., Harris A.W., Snowden S.L., \& Bickert K. (1992): ApJ 397, 158

Pildis R.A., \& McGaugh S.S. (1996): ApJL 470, 77

Sidher S.D., Sumner T.J., Quenby J.J., \& Gambhir M. (1996): A\&A 305, 308

Sidher S.D., Sumner T.J., \& Quenby J.J. (1997): In preparation

Snowden S.L., Mebold U., Hirth W., Herbstmeier U., \& Schmitt J.H.M.M. (1991): Science 252, 1529

Soltan A.M., Hasinger G., Egger R., Snowden S., \& Trümper J. (1996): A\&A 320, 685

Sumner T.J., Sidher S.D., \& Quenby J.J. (1997): In preparation

Suto Y., Makishima K., Ishisaki Y., \& Ogasaka Y. (1996): ApJL 461, 33 